\title{
RELATIONSHIP BETWEEN CHARACTERISTICS OF TEAT DUCT AND PREVALENCE OF INTRAMAMMARY INFECTIONS IN RESPECTIVE QUARTERS
}

\author{
U. Falkenberg, B.-A. Tenhagen, W. Heuwieser \\ Clinic for Reproduction, Section of Production Medicine and Quality Management, \\ Free University of Berlin, Germany
}

www.bestandsbetreuung.de

The objective of the study was to determine the relationship between morphology of the surface of teat duct particularly the level of its ceratosis and the prevalence of intramammary infections (IMI). The study was conducted on a commercial dairy herd housing about 3000 lactating dairy cows. We examined a set of cows (972 quarters) in the middle of lactation. Duplicate samples of quarter foremilk were collected monthly. The bacteriological status of quarters was recorded according to the recommendations of IDF. At the same time teats were evaluated by clinical examinations. The appearance of teat skin lesions and the status of teat duct especially the existence of hyperceratosis (HC) was documented. We defined four classes of teat duct hyperceratosis: without, slight, middle and strong HC. The rate of IMI in different classes of hyperceratosis of teat duct was compared by Chi-square analysis.

Prevalences of intramammary infections were determined three times (P1, P2 and P3) during the study period. Prevalence of infection was high for $S$. aureus (P1: $5.6 \%$ vs. P2: $4.5 \%$ vs. P3: $4.3 \%$ ), Sc. agalactiae (P1: $2.7 \%$ vs. P2: $2.6 \%$ vs. P3: $2.8 \%$ ) and CNS (P1: $10.7 \%$ vs.P2: $8.8 \%$ vs. P3: 9.6 $\%)$. Furthermore we detected IMI caused by other streptococci, yeast, E. coli and mixed infections. We found a positive correlation between status of $\mathrm{HC}$ and prevalence of IMI for Sc. agalactiae. A negative correlation was found for $S$. aureus and no correlation for CNS each time of examination. At the second and third time of collecting foremilk samples the difference in rates of intramammary infection with Sc. agalactiae in quarters with middle HC (P2: 9.21\% and P3: 13.73\%) showed significant differences $(\mathrm{p}<.05)$ to groups without $\mathrm{HC}(\mathrm{P} 2: 1.56 \%$ and $\mathrm{P} 3: 1.91 \%)$ and slight $\mathrm{HC}(\mathrm{P} 2$ : $2.33 \%$ and $\mathrm{P} 3: 2.56 \%$ ).

The results of our study indicate the existence of a correlation between morphology of teat duct surface, especially regarding to Sc. agalactiae. On the one hand $\mathrm{HC}$ can cause high intramammary infection rate with $S c$. agalactiae. On the other hand it is possible that $\mathrm{HC}$ is the consequence of a quarter infection with $S c$. agalactiae. Further research is required. 